\title{
EFEITO DE ISOLADOS DE Paecilomyces lilacinus NO DESENVOLVIMENTO DE CAFEZAIS E NA POPULAÇÃO DE Meloidogyne paranaensis
}

\author{
Effect of isolates of Paecilomyces lilacinus on the development of coffee plantations \\ and on the population of Meloidogyne paranaensis
}

\author{
Marina Capparelli Cadioli' ${ }^{1}$, Débora Cristina Santiago ${ }^{2}$, Arian Derdote de Oliveira ${ }^{3}$, \\ Vanessa dos Santos Paes $^{4}$, Giovani de Oliveira Arieira ${ }^{5}$, Fernando Cesar Baida ${ }^{6}$
}

\begin{abstract}
RESUMO
Com a finalidade de diminuir as perdas causadas pelos nematóides do gênero Meloidogyne (Goeldi, 1887) na cultura do cafeeiro, dentre as diversas medidas de manejo, o controle biológico com o fungo Paecilomyces lilacinus (Thom., 1910) Samson, 1974 se destaca como uma alternativa de controle vantajosa, quer dos pontos de vista ecológico ou econômico. Assim, neste trabalho, objetivou-se avaliar a eficiência de 10 isolados de Paecilomyces lilacinus no controle de Meloidogyne paranaensis em cafeeiro (Coffea arabica L. cv. Icatú), em casa-de-vegetação. No experimento I, as mudas de cafeeiro foram transplantadas em solo onde foram, anteriormente, cultivados tomateiros para multiplicação de $M$. paranaensis mais $50 \mathrm{~g}$ de arroz colonizado com os 10 isolados. No segundo experimento, mudas de cafeeiro foram transplantadas para substrato solo e areia (1:1) juntamente com $50 \mathrm{~g}$ de arroz colonizado com os isolados. Em seguida, as mudas foram inoculadas com \pm 5000 ovos de M. paranaensis. Nos dois experimentos, após 15 dias procedeu-se aplicação por cobertura de $50 \mathrm{~g}$ dos isolados. $\mathrm{O}$ delineamento foi inteiramente casualizado com 12 tratamentos. Após 90 dias, foram feitas as avaliações. Os isolados de P. lilacinus não afetaram o diâmetro do caule de cafeeiro. No experimento I, os isolados Pae 22, 24 e 28 promoveram o crescimento dos cafeeiros; todos os isolados reduziram a população de ovos no sistema radicular; e os isolados Pae 3 e 12 reduziram a população de $\mathrm{J} 2$ de $M$. paranaensis no solo. No experimento II, os isolados Pae 03, 10, 12 e 13 favoreceram o crescimento das plantas, mas reduziram o peso fresco do sistema radicular; todos os isolados reduziram a população de $\mathbf{J} 2$ no solo; e os isolados Pae 3, 10, 13, 18, 22 e 24 reduziram as malformações causadas por M. paranaensis nas raízes.
\end{abstract}

Termos para indexação: Controle Biológico. Paecilomyces lilacinus. Meloidogyne paranaensis.

\section{ABSTRACT}

In order to reduce the losses caused by nematodes of the genus Meloidogyne (Goeldi, 1887) in coffee plantation, among several management measures, biological control with the fungus Paecilomyces lilacinus (Thom., 1910) Samson, 1974 stands out as an advantageous alternative of control, from the ecological or economy points of view. Thus, the objective of this work was to evaluate ten isolates of Paecilomyces lilacinus in the control of Meloidogyne paranaensis in coffee trees (Coffea arabica L.) cv. Icatú, in greenhouse conditions. In the first experiment, the coffee seedlings were transplanted to substrate where tomato plants were previously cultivated, for $M$. paranaensis multiplication. The soil was mixed with $50 \mathrm{~g}$ rice colonized with ten isolates of $P$. lilacinus. In the second experiment, coffee seedlings were transplanted to substrate ( 1 sand: 1 dirt) with $50 \mathrm{~g}$ rice colonized with the isolates of $P$. lilacinus. Then, the seedlings were inoculated with suspension of $\pm 5000 \mathrm{eggs}$ of M. paranaensis. In the two experiments, a new application was made with $50 \mathrm{~g}$ of the isolates after fifteen days. The design was completely randomized with twelve treatments and ten replicates. After 90 they days were evaluated. The isolated of $P$. lilacinus did not affect the diameter of the coffee stalk. In the first experiment, the isolates Pae 22, 24, and 28 promoted the growth of the seedlings, all of the isolates reduced the population of eggs in the root system, and the isolates Pae 3 and 12 reduced the population of $\mathbf{J} 2$ of $M$. paranaensis in the soil. In the second experiment, the isolates Pae 3, 10, 12 , and 13 favored the growth of the plants but reduced the weight of the fresh roots, all of the isolates reduced the population of $\mathrm{J} 2$ in the soil, and the isolates Pae 3, 10, 13, 18, 22, and 24 reduced the malformations caused by $M$. paranaensis roots.

Index terms: Biological control, Paecilomyces lilacinus, Meloidogyne paranaensis

(Recebido em 22 de abril de 2008 e aprovado em 6 de outubro de 2008)

\footnotetext{
'Engenheira Agrônoma, Mestre, Doutoranda - Departamento de Agronomia/AGR - Universidade Estadual de Londrina/UEL - Rod. Celso Garcia Cid, Pr 445, Km 380, Bairro, Campus Universitário - Cx. P. 6001 - 86055-900 - Londrina,PR - marinacadioli@hotmail.com

Engenheira Agrônoma, Doutora - Departamento de Agronomia/AGR - Universidade Estadual de Londrina/UEL - Rod. Celso Garcia Cid, Pr 445, Km 380, Bairro, Campus Universitário - Cx. P.6001-86055-900 - Londrina,PR - santiago@uel.br

${ }^{3}$ Graduando em Agronomia - Departamento de Agronomia/AGR - Universidade Estadual de Londrina/UEL - Rod. Celso Garcia Cid, Pr 445, Km 380, Bairro, Campus Universitário - Cx. P. 6001-86055-900 - Londrina,PR - derdote@yahoo.com.br

${ }^{4}$ Graduando em Agronomia - Departamento de Agronomia/AGR - Universidade Estadual de Londrina/UEL - Rod. Celso Garcia Cid, Pr 445, Km 380, Bairro, Campus Universitário - Cx. P. 6001-86055-900 - Londrina,PR - nessinhaagro@yahoo.com.br

${ }^{5}$ Graduando em Agronomia - Departamento de Agronomia/AGR - Universidade Estadual de Londrina/UEL - Rod. Celso Garcia Cid, Pr 445, Km 380, Bairro, Campus Universitário - Cx. P. 6001 - 86055-900 - Londrina,PR - giovaniarieira@yahoo.com.br

${ }^{6}$ Engenheiro Agrônomo, Mestrando - Departamento de Agronomia/AGR - Universidade Estadual de Londrina/UEL - Rod. C elso Garcia Cid, Pr 445, Km 380, Bairro, Campus Universitário - Cx. P. 6001 - 86055-900 - Londrina,PR - fbaida@hotmail.com
} 


\section{INTRODUÇÃO}

Para diminuir as perdas das lavouras de café em razão de nematóides e reduzir para níveis mínimos os danos causados ao ambiente, com a utilização de produtos químicos, o controle biológico vem sendo considerado uma das alternativas, dentro de uma abordagem integrada, na qual se busca assegurar o desenvolvimento sustentável da agricultura. Assim, o uso de inimigos naturais é promissor e torna-se um fascinante campo de investigação, sendo potencialmente útil dentro das medidas duráveis (Stirling, 1991), podendo reduzir populações de fitonematóides para limiares abaixo do nível de dano econômico (Duncan, 1991).

Paecilomyces lilacinus (Thom., 1910) Samson, 1974, é um fungo de solo que tem se mostrado efetivo no biocontrole de espécies do gênero Meloidogyne Goeldi, 1887 (Kerry, 1990). Caracteriza-se por penetrar os ovos de nematóides, destruindo o embrião, podendo exercer forte pressão na capacidade reprodutiva das fêmeas que são colonizadas e, posteriormente, mortas (Dunn et al., 1982). No Brasil, existem registros de $P$. lilacinus em diferentes tipos de solo, cultivados ou não, em profundidades variáveis de 0-40 cm ou mais (Carneiro, 1986).

Esse fungo apresenta distribuição cosmopolita e maior frequência em solos agricultáveis (Domsch et al., 1980), tendo sido frequentemente isolado a partir de diferentes hospedeiros ou de substratos provenientes de várias localidades (Sosa-Gomez, 2002). Costa et al. (1997), estudando a associação de fungos a cistos de Heterodera glycines (Ichinohe, 1952), encontraram P. lilacinus nos municípios de Iraí de Minas - MG e Chapadão do Céu - GO. Santiago et al. (2006) obtiveram 37 isolados de $P$. lilacinus em amostras de solo oriundas de 19 municípios, distribuídos nos Estados de Maranhão, Mato Grosso do Sul, Mato Grosso, Pará, Paraná, Rio Grande do Sul e São Paulo.

Estudos envolvendo a seleção de isolados de $P$. lilacinus para o controle de nematóides são importantes na busca de microrganismos eficientes e adaptados às diferentes regiões. Freitas et al. (1999), comparando a eficiência do parasitismo de 19 isolados de P. lilacinus, observaram que $100 \%$ dos ovos de Meloidogyne javanica (Treub, 1885) estavam parasitados com isolados originários da Itália e do Peru e cerca de $70 \%$ com o isolado da França. Já o percentual de ovos parasitados pelos isolados brasileiros variou de 2 a $69 \%$. Posteriormente, Freitas et al. (1999) obtiveram sucesso na proteção de mudas de tomateiro, em casa-de-vegetação, contra $M$. javanica, por meio da incorporação de $P$. lilacinus ao substrato.
Entretanto, estudos anteriores realizados por Novaretti et al. (1986), na cultura de cana-de-açúcar, por Hewlett et al. (1988), em tabaco, e por Carneiro \& Cayrol (1991), em tomateiro, contestaram a eficiência de isolados do $P$. lilacinus como agentes de controle em condições de campo. Resultados contrários podem ocorrer, por vezes, decorrentes da inadequação dos métodos de produção de conídios do fungo, inadequação dos métodos de aplicação e avaliação dos ensaios (Kerry, 1990), e não adaptação do isolado a diferentes condições e tipos de solo (Carneiro, 1992), entre outros.

Embora resultados encorajadores sejam observados em condições brasileiras (Carneiro \& Gomes, 1993; Costa \& Campos, 1997; Freitas et al., 1999; Mizobutsi et al., 2000; Santiago et al., 2006), informações básicas sobre o comportamento de $P$. lilacinus como parasita das diferentes espécies do gênero Meloidogyne são necessárias para que seu emprego na agricultura seja recomendado.

Dessa forma, conduziu-se este trabalho, com o objetivo de avaliar o efeito de isolados de P. lilacinus, obtidos na região de Londrina-PR, no desenvolvimento do cafeeiro e na população de Meloidogyne paranaensis e selecionar os melhores isolados.

\section{MATERIALE MÉTODOS}

A eficiência dos isolados de P. lilacinus no controle de M. paranaensis em cafeeiro (C. arabica cv. Icatú) foi realizada em dois experimentos, conduzidos em casa-devegetação, em delineamento experimental inteiramente casualizado com 12 tratamentos, constituídos por 10 isolados de $P$. lilacinus, uma testemunha não tratada com $P$. lilacinus e não inoculada com $M$. paranaensis e uma testemunha apenas inoculada com $M$. paranaensis, distribuídos em dez repetições.

Os isolados de P. lilacinus avaliados pertencem à coleção de microrganismos do Laboratório de Fitopatologia da Universidade Estadual de Londrina, os quais foram obtidos em áreas de cultivo de cafeeiro na região de Londrina - PR, a exceção do isolado Pae 03 obtido em área de milho. Estes foram previamente selecionados quanto à capacidade de parasitar ovos de M. paranaensis "in vitro", em diferentes temperaturas (Cadioli et al., 2007).

Para multiplicação dos isolados de $P$. lilacinus, foram utilizados grãos de arroz parboilizados distribuídos em porções de $100 \mathrm{~g}$, juntamente com $100 \mathrm{~mL}$ de água, em embalagens de polipropileno com capacidade para $1 \mathrm{~kg}$, que foram autoclavadas durante 1 hora a $120^{\circ} \mathrm{C}$ e $1 \mathrm{~atm}$. Após o resfriamento, em cada embalagem repicaram-se três discos de $5 \mathrm{~mm}$ de diâmetro retirados das colônias de $P$. 
lilacinus desenvolvidas em BDA por sete dias. Após a repicagem, as embalagens foram deixadas em temperatura ambiente durante 15 dias para que o fungo colonizasse o substrato.

M. paranaensis foi multiplicada em plantas de tomateiro, Solanum lycopersicum L. cv. Santa Cruz, a partir das quais procedeu-se à extração dos ovos, segundo a metodologia de Boneti \& Ferraz (1981), obtendo-se uma suspensão ajustada para concentração média de 5.000 ovos e eventuais juvenis.mL $\mathrm{mL}^{-1}$.

No Experimento I, antes do cultivo das mudas de cafeeiro, plântulas de tomateiro cv. Santa Cruz, com 16 dias de idade, foram transplantadas para vasos de plástico de 1,5 $\mathrm{L}$ contendo como substrato solo e areia lavada na proporção de 1:1 $\left(\mathrm{v} \cdot \mathrm{v}^{-1}\right)$, previamente tratados com brometo de metila $\left(150 \mathrm{~mL} \cdot \mathrm{m}^{-3}\right)$. Aos 15 dias do transplantio, as plântulas foram inoculadas na região da rizosfera com $5 \mathrm{~mL}$ da suspensão, contendo \pm 5000 ovos e/ou juvenis de M. paranaensis. Essas plantas foram mantidas durante 45 dias, em casa-devegetação, para permitir a multiplicação dos nematóides e a produção da segunda geração de ovos, uma vez que $P$. lilacinus tem preferência pelo parasitismo destes.

Decorrido esse período, as plantas e o substrato foram removidos dos vasos e a parte aérea foi descartada. As raízes foram cortadas e misturadas ao mesmo substrato, ao qual procedeu-se a aplicação dos tratamentos com os isolados de $P$. lilacinus por meio da adição e mistura de $50 \mathrm{~g}$ de arroz colonizado ( $10^{9}$ esporos do fungo. $\mathrm{g}^{-1}$ de arroz). $\mathrm{O}$ substrato formado, agora, pela mistura de raízes infectadas por M. paranaensis e de grãos de arroz colonizados por $P$. lilacinus foi distribuído em sacos de polietileno com capacidade para 2,0 L. Na sequência, efetuou-se o transplantio de uma muda de cafeeiro cv. Icatú por saquinho. Nas testemunhas não tratadas com $P$. lilacinus e não inoculadas $\operatorname{com} M$. paranaensis foram introduzidas apenas 50 gramas de arroz não colonizado. Já nas testemunhas não tratadas com P. lilacinus e inoculadas com o nematóide foram introduzidas 50 gramas de arroz não colonizado mais as raízes contendo inóculo de $M$. paranaensis.

No Experimento II, as mudas de cafeeiro cv. Icatú foram transplantadas diretamente para sacos de polietileno com capacidade para 2,0 L, contendo como substrato solo e areia lavada, previamente tratados com brometo de metila $\left(150 \mathrm{~mL} \cdot \mathrm{m}^{-3}\right)$, na proporção de 1:1 $\left(\mathrm{v} . \mathrm{v}^{-1}\right)$, mais os isolados de P. lilacinus aplicados por meio da adição e mistura de $50 \mathrm{~g}$ de arroz colonizado ( $10^{9}$ esporos do fungo. $\mathrm{g}^{-1}$ de arroz). Nas testemunhas não tratadas com o fungo e não inoculadas foram introduzidos apenas 50 gramas de arroz não colonizado. Já nas testemunhas não tratadas e inoculadas com $M$. paranaensis foram introduzidas 50 gramas de arroz não colonizado mais $5 \mathrm{~mL}$ da suspensão contendo \pm 5000 ovos e eventuais juvenis de $M$. paranaensis.

Em ambos os experimentos, uma nova aplicação dos tratamentos com os isolados em cobertura foi realizada aos 15 dias do transplantio das mudas de cafeeiro. As práticas de adubação, irrigação e tratos culturais foram efetuadas de acordo com as recomendações para produção de mudas de cafeeiro.

Nos experimentos I e II, as avaliações foram realizadas aos 90 dias da primeira inoculação do fungo, sendo determinados: altura de plantas, tomada do colo até o ápice; diâmetro do caule, a $2 \mathrm{~cm}$ do colo da planta; pesos da matéria fresca da parte aérea e do sistema radicular; número de ovos por sistema radicular (Boneti \& Ferraz, 1981); números de juvenis em $250 \mathrm{cc}$ de solo (Jenkins, 1964); sobrevivência dos isolados ao final do experimento. No Experimento II, as raízes foram avaliadas quanto à presença de malformações em função da dificuldade de encontrar galhas definidas nas raízes.

Para avaliar a sobrevivência dos fungos no solo, foram coletadas, para cada isolado, amostras de $10 \mathrm{~g}$ de solo de cinco vasos por tratamento. Para cada amostra, procedeuse à diluição seriada e, da diluição $10^{-3}$, alíquotas de $1 \mathrm{~mL}$ foram distribuídas em placas de Petri contendo meio semiseletivo, em número de cinco repetições por amostra, onde no sexto dia de incubação foi determinado o percentual de desenvolvimento das colônias em cada placa.

Os dados obtidos quanto ao desenvolvimento das plantas de café foram submetidos às análises de variância e ao teste de Scott-Knott a 5\% de significância de erro, para comparação de médias. Já os dados obtidos quanto ao efeito dos isolados do fungo P. lilacinus, quanto ao número de juvenis de segundo estágio (J2), número de ovos por sistema radicular, número de malformações e percentagem de sobrevivência do fungo no solo foram transformados em $\sqrt{ } \mathrm{x}+0,5$ para análise estatística $\mathrm{e}$ submetidos ao teste de Scott-Knott a 5\% de significância de erro, para comparação das médias.

\section{RESULTADOS E DISCUSSÃO}

No experimento I, os isolados de $P$. lilacinus testados não afetaram o diâmetro do caule de cafeeiro, mas alguns deles (Pae 10, 13, 18, 22, 24 e 28) promoveram o seu crescimento (altura) e, concomitantemente, aumentaram o peso fresco da parte aérea. Contudo, o peso fresco do sistema radicular foi reduzido em $70 \%$ dos isolados testados comparados com a testemunha (Tabela 1). Os três isolados (Pae 22, 24 e 28) que não reduziram o sistema radicular do cafeeiro promoveram o seu crescimento (altura e peso da parte aérea). 
Tabela 1 - Efeito de isolados de Paecilomyces liacinus sobre o desenvolvimento das plantas de cafeeiro inoculadas com Meloidogyne paranaensis, experimento I.

\begin{tabular}{|c|c|c|c|c|}
\hline \multirow{2}{*}{ Tratamentos } & \multirow{2}{*}{ Altura (cm) } & \multirow{2}{*}{$\begin{array}{l}\text { Diâmetro do Caule } \\
\qquad(\mathrm{cm})\end{array}$} & \multicolumn{2}{|c|}{ Peso (g) } \\
\hline & & & Parte aérea & Raízes \\
\hline Pae 03 & $29,35^{1} \quad b^{2}$ & 4,44 a & $11,33 \mathrm{~b}$ & $7,96 \mathrm{~b}$ \\
\hline Pae 10 & 38,50 a & 4,82 a & $19,83 \mathrm{a}$ & $9,38 \mathrm{~b}$ \\
\hline Pae 12 & $30,40 \mathrm{~b}$ & 4,65 a & $14,26 \mathrm{~b}$ & $9,62 \mathrm{~b}$ \\
\hline Pae 13 & $36,05 \mathrm{a}$ & 4,61 a & $18,70 \mathrm{a}$ & $9,28 \mathrm{~b}$ \\
\hline Pae 18 & 38,25 a & 4,73 a & $16,97 \mathrm{a}$ & $8,96 \mathrm{~b}$ \\
\hline Pae 20 & $29,75 \mathrm{~b}$ & $4,27 \mathrm{a}$ & $13,69 \mathrm{~b}$ & $8,20 \mathrm{~b}$ \\
\hline Pae 21 & $27,95 \mathrm{~b}$ & 4,17 a & $11,48 \quad b$ & $7,66 \mathrm{~b}$ \\
\hline Pae 22 & 37,95 a & 4,51 a & 18,86 a & 10,14 a \\
\hline Pae 24 & 38,40 a & 4,94 a & $22,47 \quad a$ & $11,47 \quad \mathrm{a}$ \\
\hline Pae 28 & 35,80 a & 4,73 a & 22,41 a & 10,62 a \\
\hline Test. inoculada & $32,50 \mathrm{~b}$ & 4,84 a & $11,22 \mathrm{~b}$ & 11,53 a \\
\hline Test. não inoculada & $31,60 \mathrm{~b}$ & 4,59 a & $10,75 \mathrm{~b}$ & $11,50 \mathrm{a}$ \\
\hline $\mathrm{CV}(\%)$ & 14,96 & 15,20 & 31,30 & 24,49 \\
\hline
\end{tabular}

${ }^{1}$ Os dados são médias de 10 repetições.

${ }^{2}$ Médias seguidas de letras iguais nas colunas, não diferem entre si, pelo teste de Scott-knott em nível de 5\% de significância de erro.

No experimento I, a população de juvenis do segundo estádio (J2) de M. paranaensis no solo só foi reduzida quando os isolados Pae 3 e 12 foram inoculados no cafeeiro. Já a população de ovos por sistema radicular foi reduzida por todos os isolados testados comparados com a testemunha. Todos os isolados de P. lilacinus foram recuperados do solo ao final do experimento I, com maior sobrevivência para os isolados Pae 3, 12, 20, 21 e 22 (Tabela 2).

No experimento II, os isolados de P. lilacinus testados, também não afetaram o diâmetro do caule do cafeeiro, porém $60 \%$ deles reduziram o crescimento (altura) bem como o peso fresco de parte aérea (Pae 18, 20, 21, 22, 24 e 28). O peso fresco do sistema radicular foi reduzido na testemunha inoculada com $M$. paranaensis comparada com as não inoculadas, bem como em todos os cafeeiros inoculados com os isolados de $P$. lilacinus e $M$. paranaensis (Tabela 3 ).

A população de $\mathrm{J} 2$ de $M$. paranaensis no solo foi reduzida $(\mathrm{P} \leq 0,05)$ por todos os isolados testados. Dos 10 isolados avaliados, seis deles (Pae 3, 10, 13, 18, 22 e 24) reduziram $(\mathrm{P} \leq 0,05)$ as malformações nas raízes causadas por M. paranaensis. Todos os isolados de P. lilacinus foram recuperados do solo, evidenciando elevada taxa de sobrevivência (Tabela 4).

Quando comparados os dois experimentos, podese observar que o desenvolvimento das plantas de cafeeiro, no experimento II, foi mais afetado que o das plantas do experimento I, com menor crescimento (altura), peso fresco de parte aérea e peso fresco do sistema radicular. Esses resultados são semelhantes aos observados por Arruda (1960), o qual cita que em plantas infestadas com espécies do gênero Meloidogyne, o crescimento, a translocação de água e nutrientes e a produção do cafeeiro são seriamente comprometidos. Tal fato pode ter ocorrido pelo maior tempo de multiplicação da população de nematóides no experimento II em relação ao experimento I.

Com relação ao efeito dos isolados de $P$. lilacinus sobre os $\mathrm{J} 2$ de $M$. paranaensis no solo, no experimento I, os dados foram variáveis, verificando-se que os isolados não reduziram a população de juvenis no solo, com exceção dos isolados Pae 3 e 12. Morgan-Jones \& Rodriguez-Kábana (1995), relatam que os estádios juvenis, em função de sua mobilidade, são menos vulneráveis ao ataque pelo micélio dos fungos. La Mondia \& Brodie (1984), apontaram que ovos nos estádios iniciais do desenvolvimento embriogênico são mais facilmente parasitados do que quando possuem o juvenil de segundo estádio $\left(\mathrm{J}_{2}\right)$ já formado, o que explicaria a baixa eficiência da maioria dos isolados de $P$. lilacinus contra os $\mathrm{J} 2$ de $M$. paranaensis no solo. No experimento II, entretanto, observou-se que os isolados Pae 18 e 22 reduziram o número de $\mathrm{J} 2$ no solo. 
Tabela 2 - Efeito de isolados de Paecilomyces liacinus na população de ovos e juvenis de segundo estádio (J2) de Meloidogyne paranaensis no solo e na sobrevivência de isolados no solo do experimento I.

\begin{tabular}{|c|c|c|c|}
\hline Tratamentos & $\begin{array}{l}\mathrm{N}^{\mathrm{o}} \text { de } \mathrm{J} 2 \text { por } 250 \mathrm{cc} \text { de } \\
\text { solo }\end{array}$ & $\begin{array}{c}\mathrm{N}^{\circ} \text { de Ovos por sistema } \\
\text { radicular }\end{array}$ & Sobrevivência (\%) \\
\hline Pae 03 & $166,67^{1} \mathrm{j}^{3}$ & $464,69^{1} d$ & $58,00^{2}$ a \\
\hline Pae 10 & $1013,33 \mathrm{~g}$ & $1270,50 \mathrm{c}$ & $32,00 \mathrm{~b}$ \\
\hline Pae 12 & $246,67 \mathrm{i}$ & $1542,50 \mathrm{c}$ & 62,00 a \\
\hline Pae 13 & $1500,00 \mathrm{f}$ & $709,32 \mathrm{c}$ & $40,00 \mathrm{~b}$ \\
\hline Pae 18 & $2446,67 \mathrm{c}$ & $992,04 \mathrm{c}$ & $16,00 \mathrm{c}$ \\
\hline Pae 20 & $1600,00 \mathrm{e}$ & $316,40 \mathrm{~d}$ & 50,00 a \\
\hline Pae 21 & $1733,33 \mathrm{~d}$ & $542,50 \mathrm{~d}$ & 47,00 a \\
\hline Pae 22 & $3280,00 \mathrm{~b}$ & $507,08 \mathrm{~d}$ & 66,00 a \\
\hline Pae 24 & $633,33 \mathrm{e}$ & $1247,88 \mathrm{c}$ & $19,00 \mathrm{c}$ \\
\hline Pae 28 & 3893,33 a & 2742,24 b & $8,00 \mathrm{~d}$ \\
\hline Test. inoculada & $573,33 \mathrm{~h}$ & 5982,50 a & 0,00 \\
\hline Test. não inoculada & $0,00 \mathrm{k}$ & $0,00 \mathrm{e}$ & $0,00 \mathrm{e}$ \\
\hline $\mathrm{CV}(\%)$ & 2,86 & 72,28 & 17,65 \\
\hline
\end{tabular}

${ }^{1}$ Os dados são médias de 10 repetições e foram transformados em $\sqrt{ } \mathrm{x}+0,5$ para análise estatística.

${ }^{2}$ Os dados são médias de 25 repetições e foram transformados em $\sqrt{ } \mathrm{x}+0,5$ para análise estatística.

${ }^{3}$ Médias seguidas de letras iguais nas colunas, não diferem entre si, pelo teste de Scott-knott em nível de 5\% de significância de erro.

Tabela 3 - Efeito dos isolados de Paecilomyces liacinus sobre o desenvolvimento das plantas de cafeeiro inoculadas com Meloidogyne paranaensis, experimento II.

\begin{tabular}{|c|c|c|c|c|}
\hline \multirow{2}{*}{ Tratamentos } & \multirow{2}{*}{ Altura $(\mathrm{cm})$} & \multirow{2}{*}{$\begin{array}{l}\text { Diâmetro do Caule } \\
\qquad(\mathrm{cm})\end{array}$} & \multicolumn{2}{|c|}{ Peso (g) } \\
\hline & & & Parte aérea & Raízes \\
\hline Pae 03 & $40,40^{1} a^{2}$ & 5,04 a & 23,85 a & $16,80 \mathrm{~b}$ \\
\hline Pae 10 & 38,40 a & 4,76 a & 25,64 a & $19,17 \mathrm{~b}$ \\
\hline Pae 12 & $40,65 \mathrm{a}$ & 5,54 a & 23,55 a & $17,28 \mathrm{~b}$ \\
\hline Pae 13 & $36,90 \mathrm{a}$ & 4,73 a & $18,69 \mathrm{~b}$ & $11,47 \mathrm{c}$ \\
\hline Pae 18 & $32,70 \quad b$ & 4,14 a & $11,83 \mathrm{~b}$ & $10,45 \mathrm{c}$ \\
\hline Pae 20 & $34,15 \mathrm{~b}$ & 4,84 a & $18,64 \mathrm{~b}$ & $14,55 \mathrm{~b}$ \\
\hline Pae 21 & $33,22 \mathrm{~b}$ & 4,98 a & $19,72 \mathrm{~b}$ & $14,72 \mathrm{~b}$ \\
\hline Pae 22 & $35,50 \mathrm{~b}$ & 4,71 a & $18,87 \quad b$ & $11,71 \mathrm{c}$ \\
\hline Pae 24 & $35,80 \mathrm{~b}$ & 4,47 a & $19,49 \mathrm{~b}$ & $16,62 \mathrm{~b}$ \\
\hline Pae 28 & $32,40 \quad b$ & 4,68 a & $13,61 \quad b$ & $8,58 \mathrm{c}$ \\
\hline Test. inoculada & $40,20 \mathrm{a}$ & $4,93 \mathrm{a}$ & 24,49 a & $19,69 \mathrm{~b}$ \\
\hline Test não inoculada & 39,00 a & 5,68 a & 26,83 a & 30,03 a \\
\hline $\mathrm{CV}(\%)$ & 16,97 & 19,53 & 42,65 & 41,63 \\
\hline
\end{tabular}

${ }^{1}$ Os dados são médias de 10 repetições

${ }^{2}$ Médias seguidas de letras iguais nas colunas, não diferem entre si, pelo teste de Scott-knott em nível de 5\% de significância de erro. 
Tabela 4 - Efeito de isolados de Paecilomyces lilacinus na população de juvenis do segundo estádio (J2) no solo, malformações causadas por Meloidogyne paranaensis nas raízes e na sobrevivência dos isolados no solo do experimento II.

\begin{tabular}{|c|c|c|c|}
\hline Tratamentos & $\begin{array}{c}\mathrm{N}^{\mathrm{o}} \text { de } \mathrm{J} 2 \text { por } \\
250 \text { cc de solo }\end{array}$ & $\begin{array}{c}\mathrm{N}^{\circ} \text { de malformações } \\
\text { nas raízes }\end{array}$ & $\begin{array}{c}\text { Sobrevivência } \\
(\%)\end{array}$ \\
\hline Pae 03 & $513,33^{1} \mathrm{f}^{3}$ & $18,70^{1} \quad \mathrm{~B}$ & $52,00^{2} \quad \mathrm{~d}$ \\
\hline Pae 10 & 820,00 e & $29,40 \quad \mathrm{~B}$ & $69,00 \mathrm{c}$ \\
\hline Pae 12 & 1386,67 & $50,80 \mathrm{~A}$ & 93,00 a \\
\hline Pae 13 & $426,67 \mathrm{f}$ & $22,70 \quad \mathrm{~B}$ & 83,00 b \\
\hline Pae 18 & 133,33 & $19,90 \quad \mathrm{~B}$ & $79,00 \mathrm{~b}$ \\
\hline Pae 20 & $1033,33 \mathrm{~d}$ & $48,80 \mathrm{~A}$ & $56,00 \mathrm{~d}$ \\
\hline Pae 21 & $453,33 \mathrm{f}$ & $45,00 \mathrm{~A}$ & $52,00 \mathrm{~d}$ \\
\hline Pae 22 & $193,33 \mathrm{~g}$ & $31,70 \quad \mathrm{~B}$ & $93,00 \quad \mathrm{a}$ \\
\hline Pae 24 & $433,33 \mathrm{f}$ & $30,50 \mathrm{~B}$ & $48,00 \mathrm{~d}$ \\
\hline Pae 28 & $2086,67 \quad b$ & $40,10 \mathrm{~A}$ & $56,00 \mathrm{~d}$ \\
\hline Test. inoculada & 2653,33 a & $49,30 \mathrm{~A}$ & $0,00 \mathrm{e}$ \\
\hline Test. não inoculada & $0,00 \mathrm{~h}$ & $0,00 \quad \mathrm{C}$ & $0,00 \mathrm{e}$ \\
\hline $\mathrm{CV}(\%)$ & 10,08 & 46,98 & 12,36 \\
\hline
\end{tabular}

${ }^{1}$ Os dados são médias de 10 repetições e foram transformados em $\sqrt{ } \mathrm{x}+0,5$ para análise estatística.

${ }^{2}$ Os dados são médias de 25 repetições e foram transformados em $\sqrt{ } \mathrm{x}+0,5$ para análise estatística.

${ }^{3}$ Médias seguidas de letras iguais nas colunas, não diferem entre si, pelo teste de Scott-knott em nível de 5\% de significância de erro.

Quanto ao número de ovos de nematóides, todos os isolados mostraram capacidade na redução destes nas raízes em comparação com a testemunha no experimento I, especialmente os isolados Pae 3, 20, 21 e 22. Resultados semelhantes foram verificados por Cabanillas et al. (1989) e Freitas et al. (1999), trabalhando com as espécies Meloidogyne incognita (Kofoid \& White, 1919) e $M$. javanica em tomateiros tratados com diferentes isolados de $P$. lilacinus.

No experimento II, seis dos 10 isolados de $P$. lilacinus reduziram as malformações causadas por $M$. paranaensis nas raízes dos cafeeiros. Resultados semelhantes foram encontrados por Jatala (1985), o qual observou que $P$. lilacinus foi capaz de reduzir o número de galhas de $M$. incognita em raízes de tomateiro. Essa diminuição pode ser motivada pelo parasitismo preferencial de ovos pelo fungo, ocasionando a morte dos embriões, o que resulta em menor número de juvenis infectantes (Jatala, 1986)

Já, quanto a sobrevivência do fungo $P$. lilacinus no solo, ao final dos dois experimentos, notou-se que todos os isolados foram recuperados do solo com melhor sobrevivência para o isolado Pae 22 nos dois experimentos. Godoy et al. (1983), também, observaram que os isolados de $P$. lilacinus além de eficientes na redução da infestação de $M$. arenaria em abóbora (Cucurbita maxima Duch) apresentaram elevada capacidade de colonização e sobrevivência no solo em condições de casa-de-vegetação.

\section{CONCLUSÕES}

Os isolados de $P$. lilacinus não afetaram o diâmetro do caule de cafeeiro. No experimento I, os isolados Pae 22, 24 e 28 não reduziram o peso do sistema radicular dos cafeeiros e promoveram o seu crescimento. A população de $\mathrm{J} 2$ de $M$. paranaensis no solo foi reduzida pelos isolados Pae 3 e 12. Todos os isolados reduziram a população de ovos no sistema radicular. Os isolados Pae 3, 12, 20, 21 e 22 apresentaram maior sobrevivência ao final desse experimento.

No experimento II, os isolados Pae 03, 10, 12 e 13 favoreceram o crescimento (altura) bem como o peso fresco de parte aérea. Todos os isolados reduziram o peso fresco do sistema radicular. A população de $\mathrm{J} 2$ no solo foi reduzida por todos os isolados. Os isolados Pae 3, 10, 13, 18, 22 e 24 reduziram as malformações causadas por $M$. paranaensis nas raízes. Os isolados de $P$. lilacinus foram recuperados do solo em percentagem superior ao experimento I. 


\section{REFERÊNCIAS BIBLIOGRÁFICAS}

ARRUDA, H.V. de. Redução no crescimento de cafeeiro com um ano de campo, devido ao parasitismo de nematóides. Bragantia, Campinas, v.19, p.179-182, 1960.

BONETI, J.I.S.; FERRAZ, S. Modificação do método de Hussey e Barker para a extração de ovos de Meloidogyne exigua de raízes de cafeeiro. Fitopatologia Brasileira, Brasília, v.6, n.3, p.533, 1981.

CABANILLAS, E.; BARRER, K.R.; NELSON, L.A. Growth of isolates of Paecilomyces lilacinus on their efficacy in biocontrol of Meloidogyne incognita on tomato. Journal of Nematology, Lakeland, v.21, n.2, p.164-172, 1989.

CADIOLI, M.C.; SANTIAGO, D.C.; HOSHINO, A.D.; HOMECHIN, M. Crescimento micelial e parasitismo de Paecilomyces lilacinus sobre ovos de Meloidogyne paranaensis em diferentes temperaturas "in vitro". Ciência e Agrotecnologia, Lavras, v.31, n.2, p.305-311, mar./abr. 2007.

CARNEIRO, R.M.D.G. Estude des possibilities d'utilisation du champignon nematophage

Paecilomyces lilacinus (Thom.) Samson, 1974, comme agent de lutte biologique contre meloidogyne arenaria (Neal, 1889), Chitwood, 1949. 1986. 119f. Tese (Doutorado)-Universite des Sciences et Techniques du Languedoc, Paris.

CARNEIRO, R.M.D.G. Princípios e tendências do controle biológico de nematóides com fungos nematófagos. Pesquisa Agropecuária Brasileira, Brasília, v.27, p.113-121, 1992.

CARNEIRO, R.M.D.G.; CAYROL, J.C. Relationship between inoculum density of the nematophagus fungus Paecilomyces lilacinus and control of Meloidogyne arenaria on tomato. Revue Nematologie, v.14, n.4, p.629-634, 1991.

CARNEIRO, R.M.D.G.; GOMES, C.B. Metodologia e teste de patogenicidade de Paecilomyces lilacinus e $P$. fumosoroseus em ovos de Meloidogyne javanica. Nematologia Brasileira, Piracicaba, v.7, n.1, p.66-75, 1993.

COSTA, S.B.; CAMPOS, V.P. Obtenção de Fêmeas de Heterodera glycines em hidroponia e testes de patogenicidade de fungos isolados de cistos a fêmeas de H. glycines e de Meloidogyne spp. Summa Phytopathologica, Piracicaba, v.23, p.239-243, 1997.

COSTA, S.B.; CAMPOS, V.P.; MENEZES, M. Fungos associados a cistos de Heterodera glycines no Brasil. Nematologia Brasileira, Piracicaba, v.21, n.2, p.31-37, 1997.

DOMSCH, K.H.; GAMS, W.; ANDERSON, T.H. Compedium of Soil Fungi. New York: Academic, 1980. v.1.

DUNCAN, L.W. Current options for nematode management. Annual Review of Phytopathology, v.29, p.469-490, 1991.

DUNN, M.T.; SAYRE, R.M.; CANELL, A.; WERGIN, W.P. Colonization of nematode eggs by Paecilomyces lilacinus (Thom) Samson as observed with scanning electron microscope. Scanning Electron Microscopy, p.1351-1357, 1982.

FREITAS, L.G.; FERRAZ, S.; ALMEIDA, A.M.S. Controle de Meloidogyne javanica em tomateiro pela produção de mudas e substrato infestado com Paecilomyces lilacinus. Nematologia Brasileira, Brasília, v.23, n.1, p.65-73, 1999.

GODOY, G.; RODRIGUEZ-KÁBANA, R.; MORGANJONES, G. Fungal parasites of Meloidogyne arenaria eggs in Alabama soil. A mycological survey and greenhouse studies. Nematropica, v.13, p.207-213, 1983.

HEWLETT, T.E.; DICKSON, D.W.; MITCHELL, M.E. Evaluation of Paecilomyces lilacinus as a biocontrol agent of Meloidogyne javanica on tobacco. Journal of Nematology, v.20, n.4, p.578-584, 1988.

JATALA, P. Biological control of nematodes. In: SASSER, J.N.; CARTER, E.C. An advanced treatise on Meloidogyne. North Carolina: North Carolina State University, 1985. p.303-308.

JATALA, P. Biological control of plant-parasitic nematodes. Annual Review of Phytopathology, v.24, p.453-489, 1986.

JENKINS, W.R. A rapid centrifugal-flotation technique for separating nematodes from soil. Plant Disease Reporter, v.48, p.692, 1964. 
KERRY, B.R. An assessment of progress toward microbial controle of plant parasitic nematode. Journal of Nematology, v.22, n.45, p.621-631, 1990. Supplement.

LA MONDIA, J.A.; BRODIE, B.B. An observation chamber techinique for evaluating potencial biocontrol agents of Globodera rostochiensis. Journal of Nematology, v.16, n.1, p.112-115, 1984.

MIZOBUTSI, E.H.; FERRAZ, S.; RIBEIRO, R.C.F. Avaliação do parasitismo de diversos isolados fúngicos em ovos de Heterodera glycines e Meloidogyne javanica. Nematologia Brasileira, Brasília, v.24, n.2, p.167-172, 2000.

MORGAN-JONES, G.; RODRIGUEZ-KÁBANA, R. Phytonematode pathology fungal modes of action: a perspective. Nematropica, v.15, p.107-114, 1985.
NOVARETTI, W.R.T.; DINARDO-MIRANDA, L.L.; TOTINO, L.C.; STRABELLI, J. Efeito da aplicação conjunta do fungo Paecilomyces lilacinus e do nematicida Furadan 5 G no controle de nematóides em cana-de-açúcar. Nematologia Brasileira, Piracicaba, v.10, p.133-144, 1986.

SANTIAGO, D.C.; HOMECHIN, M.; SILVA, J.F.V.; RIBEIRO, E.R.; GOMES, B.C.; SANTORO, P.H. Seleção de isolados de Paecilomyces lilacinus (Thom.) Samson para controle de Meloidogyne paranaensis em tomateiro. Ciência Rural, Santa Maria, v.36, n.4, p.1055-1064, 2006.

SOSA-GOMEZ, D.R. Fungos entomopatogênicos: catálogo de isolados. Londrina: Embrapa Soja, 2002. v.1. (Série documentos).

STIRLING, G.R. Biological control of plant parasitic nematodes. Wallingford: CAB International, 1991. 282p. 